\title{
Research on Construction Risk Evaluation of EPC Project of Transmission and Distribution Engineering Based on Design Enterprise
}

\author{
Zhao Zhongze ${ }^{1}$, Meng Qichen ${ }^{2}$ and Pang Nansheng ${ }^{2 *}$ \\ ${ }^{1}$ Zhangjiakou Power Supply Company, State Grid Jibei Electric Power Company Limited, Zhangjiakou, Hebei, 075001, China \\ ${ }^{2}$ School of Economics and Management, North China Electric Power University, Beijing, 102206, China
}

\begin{abstract}
The EPC general contracting model of power transmission and transformation projects consisting of design, procurement and construction has gradually been favored by the owners in recent years. Among the EPC general contractors, design institutes often occupy a leading position by virtue of their design advantages, but they are inferior in construction management, and there are many construction risk factors. In this paper, the potential risks in the construction phase are stripped out, combined with the characteristics of power transmission and transformation projects, the risk identification system is established, based on the gray whitening weight function, the fuzzy comprehensive evaluation method is used to evaluate the construction risk of the EPC project of the power transmission and transformation project led by the design enterprise.
\end{abstract}

\section{Introduction}

Generally speaking, the static investment required for power transmission and transformation projects is huge, and the construction period and operation and maintenance cycle of the project are long. The EPC general contractor, which is led by the design enterprise, needs to manage the whole process. However, because the general contractor lacks the construction team and construction management experience, the management of the construction phase is often perfunctory, and it is easy to deviate from the design expectations and affect the normal operation of the project. Therefore, in order to maximize the role of the EPC general contractor, which is led by the design enterprise, in the power transmission and transformation project, it is necessary to strengthen its ability to control the construction risk.

At present, China has made very useful results for the risk research of power transmission and transformation engineering and EPC mode. Li Kang took the management of foreign power transmission and transformation projects as the research object, and analyzed the general trend of the risk of the international power transmission and transformation market from various angles [1]. Yan Han used the expert scoring method and the RPN risk importance method to quantitatively analyze the project risk [2]. Lin Zhiming combined with the characteristics of power transmission and transformation engineering, built a management process system model, using fuzzy comprehensive evaluation method to analyze the evaluation indicators
[3]. Zhang Limin clarified the five principles of dividing the owner management interface, and gave the specific implementation steps under the EPC mode [4]. Zhang Canjiang expounded the risks of each stage through the analysis of the characteristics of EPC mode and power transmission and transformation engineering [5]. Zhong Ying analyzed the characteristics and potential risks of the EPC model and proposed improved and improved opinions [6]. Lan Bo et al. introduced the specific performance of EPC mode in power transmission and transformation projects from five aspects: project cost, personnel cost, own risk, planned construction period and construction quality [7]. Xie Pengfei used SWOT analysis to demonstrate the feasibility of EPC mode in power transmission and transformation projects [8]. However, in the EPC model risk research of various power transmission and transformation projects, the general contracting with the design enterprise as the leader is not perfect in the construction stage, and further exploration is needed.

This paper considers that the general contracting with the design enterprise as the main body has too many uncertain factors in the construction stage of the project, which makes the contractor in a passive position. Therefore, this paper will focus on analyzing the risk factors at this stage, establish a risk identification system, and use fuzzy comprehensive evaluation to quantitatively analyze the uncertainty of risk, and provide reference for subsequent research work.

*Corresponding author's e-mail: pnsh@ncepu.edu.cn 


\section{Analysis of construction risk factors of power transmission and transformation projects}

In view of the cumbersome process at the construction stage, it is necessary to comprehensively analyze the construction risk factors of the EPC power transmission and transformation project led by the design enterprise under various possible conditions.

\subsection{Technical risk $U_{1}$}

- Rationality of construction drawing design and integrity risk $U_{11}$. During the design process, designers will not fully consider the current level of technical application and make designs that exceed the capabilities of the constructor. We must pay more attention to the details, to ensure that the quality of the construction drawings to a certain extent is to ensure the quality of construction.

- Design change risk $U_{12}$. Design changes will not only affect the progress of the project, but also increase the amount of engineering. In the EPC mode, the contractor and the owner sign the total price contract, and the increase in costs caused by the design change must be borne by the contractor. Therefore, the design enterprise must have a clear understanding of the line and try to reduce the losses caused by the design change.

- "Four new" technology application risks $U_{13}$. The application of "four new" technologies can improve the average level of society. However, if it is not handled properly, it will bring huge hidden dangers to the project. As a designer, we must fully consider the technical characteris-tics of the project, and make the technology new but fully grasped.

\subsection{Security risks $U_{2}$}

- Natural disaster risk $U_{21}$. The erection of power transmission and transformation engineering lines is mostly in the field, and natural factors affect the construction progress. At the design stage, the general contractor must take into account the impact of natural disasters and reduce losses.

- Construction safety risks $U_{22}$. When constructing power transmission and transformation lines, it is necessary to pay attention to the cooperation between highaltitude workers, to avoid the injury and death caused by electric shock and foundation pit collapse, and to make emergency measures.

- Site security risks $U_{23}$. When working in the field, the mobility of personnel is large, and it is necessary to ensure the safety of materials and prevent theft.
- $\quad$ Equipment safety risk $U_{24}$. In the construction process, the use and lease of equipment must strictly abide by national and industrial standards, and pay attention to protect the personal safety of construction workers.

\subsection{Material and financial risks $U_{3}$}

- Risk of material price changes $U_{31}$. Power transmission and transformation projects require a large amount of steel and cables, and the prices of these two materials have fluctuated greatly in recent years. Although the spread reserve fee is included in the budget, it is not completely avoided by the problem of rising material prices.

- Capital flow risk $U_{32}$. During the construction process, due to the shortage of funds of the owner or the general contractor, the capital chain will be broken, which will affect the progress of the project. This requires the design enterprise to have an objective and accurate understanding of its own economic strength.

- Monetary policy risk $U_{33}$. If in unstable countries and regions, we must attach great importance to the study of local monetary policy, and do not let the owner's policy to create unnecessary losses.

\subsection{Management risk $U_{4}$}

- Institutional setting risk $U_{41}$. EPC general contracting enterprises must set up a management mode of linear functional structure, under the jurisdiction of the design department, marketing department, construction department and other departments. However, the general contractor with design as the leader often only meets the professional requirements of the design department, which is a big test for the contractor's talent reserve.

- Talent risk $U_{42}$. EPC general contracting is often suitable for the management of large and medium-sized projects, not only design talents, but also complex talents who understand project management. This will avoid unnecessary losses caused by professional barriers.

- Communication risk $U_{43}$. Communication problems arising from differences in opinions, responsibilities, and resource allocation arise during work. These require the contractor to fully consider the design function, analyze the specific problem and create a good communication environment.

\subsection{Contract risk $U_{5}$}

- Risk of bidding contract $U_{51}$. Since the contractor is the leader of the design enterprise, the budget is set according to the bill of 
quantities at the time of bidding, and some provisions of the clause are unclear. Moreover, if the above four risks are not adequately controlled, then the problem will be concentrated in the acceptance stage.

- Subcontract risk $U_{52}$. Due to the contractor's own lack of understanding of these links, the inaccurate price control and the responsibilities of the responsible party often appear when the contract is concluded. These may make the contractor disadvantaged in possible economic disputes.

- Contract management risk $U_{53}$. The contractor must pay attention to the completion of the specific clauses from the start of the project to the completion of the project, and promptly communicate with the performer in case of problems, and try to avoid the situation of lost work and rework.

\section{Establishment of fuzzy comprehensive evaluation model for construction risk}

The fuzzy comprehensive evaluation method is a comprehensive evaluation of the research objects affected by many factors. The analytic hierarchy process is used to weight each factor, and the fuzzy mathematical model is used to quantitatively calculate the risk level of the research object.

\subsection{Applying AHP to empower various construction risk factors}

3.1.1 Construct a judgment matrix for each risk factor and sub-factor. This step requires a ninepoint scoring method to compare the relative importance of the two factors, giving an assessment and assignment.

3.1.2 Calculate the largest eigen value of the judgment matrix and its corresponding eigenvector. This article takes the square root method for calculation. The calculation steps are as follows:

- Calculate the product of each row element of the judgment matrix.

$$
M_{i}=\prod_{j=1}^{k} A_{i j}(I=1,2, \ldots, k)
$$

- $\quad$ Calculate the nth root of $\bar{W}_{l}$.

$$
\overline{W_{l}}=\sqrt[n]{M_{i}}
$$

- $\quad$ Normalize the vector $\bar{W}=\left[\overline{W_{1}}, \overline{W_{2}}, \ldots, \overline{W_{k}}\right]^{T}$.

$$
W_{i}=\frac{\overline{W_{l}}}{\sum_{i=1}^{n} \overline{W_{l}}}
$$

$\mathrm{W}=\left[W_{1}, W_{2}, \ldots, W_{k}\right]^{T}$ is the feature vector of the judgment matrix obtained above.
- Calculate the largest eigen value of the judgment matrix $\lambda_{\max }$.

$$
\lambda_{\max }=\sum_{i=1}^{k} \frac{(A W)_{i}}{n W_{i}}
$$

In the middle, $\lambda_{\max }-$ Matrix A maximum eigenvalue; $(A W)_{i}-$ The ith element of $A W$.

3.1.3 Consistency test on the judgment matrix. Calculation formula of consistency index $\mathrm{Cl}$.

$$
C I=\frac{\lambda_{\max }-n}{n-1}
$$

In the middle, $\mathrm{n}$ - order of the judgment matrix.

Then, by looking up the randomness index RI, the ratio $\mathrm{CI} / \mathrm{RI}$ is calculated in turn. If the ratio is less than 0.1 , it can be considered that the judgment matrix obtained by the evaluator reaches the consistency requirement; otherwise, the judgment matrix needs to be rewritten to analyze.

\subsection{Establishment of fuzzy comprehensive risk assessment model based on grey whitening weight function}

\subsubsection{Division of different risk levels}

This paper divides the project risk criteria into five levels, namely "high", "significant", "medium", "lower" and "low", and the corresponding assignment matrix is $\mathrm{M}=\left|\begin{array}{lllll}9 & 7 & 5 & 3 & 1\end{array}\right|^{\mathrm{T}}$.

\subsubsection{Establishment of gray whitening function}

- According to the division of risk in 3.2.1, the corresponding whitening weight function is defined as $f_{1}(\mathrm{x}) 、 f_{2}(x), f_{3}(x), f_{4}(x)$, $f_{5}(x)$.

$$
\begin{aligned}
& f_{1}(x)=\left\{\begin{array}{c}
\frac{1}{9} x, 0 \leq x \leq 9 \\
1,9 \leq x \leq 10
\end{array}\right. \\
& f_{2}(x)=\left\{\begin{array}{c}
\frac{1}{7} x, 0 \leq x \leq 7 \\
\frac{10-x}{3}, \quad 7 \leq x \leq 10
\end{array}\right. \\
& f_{3}(x)=\left\{\begin{array}{c}
\frac{1}{5} x, 0 \leq x \leq 5 \\
\frac{10-x}{5}, \quad 5 \leq x \leq 10
\end{array}\right. \\
& f_{4}(x)=\left\{\begin{array}{c}
\frac{1}{3} x, 0 \leq x \leq 3 \\
\frac{10-x}{7}, \quad 3 \leq x \leq 10
\end{array}\right. \\
& f_{5}(x)=\left\{\begin{array}{c}
1,0 \leq x \leq 1 \\
\frac{10-x}{9}, 1 \leq x \leq 10
\end{array}\right.
\end{aligned}
$$

- The expert group scored the risk factors at all levels and listed the risk indicator score table.

- According to the determined whitening weight function, the score table is substituted, and the 
risk index value $r_{i j}$ and the weight matrix $R_{i j}$

are calculated at each level.

$$
r_{i j}=\left(\frac{\sum f_{1}(x)}{\sum_{n=1}^{5} \sum f_{n}(x)}, \frac{\sum f_{2}(x)}{\sum_{n=1}^{5} \sum f_{n}(x)}, \frac{\sum f_{3}(x)}{\sum_{n=1}^{5} \sum f_{n}(x)}, \frac{\sum f_{4}(x)}{\sum_{n=1}^{5} \sum f_{n}(x)}, \frac{\sum f_{5}(x)}{\sum_{n=1}^{5} \sum f_{n}(x)}\right)
$$

$$
R_{i}=\left(r_{i 1}, r_{i 2}, \ldots, r_{i j}\right)^{T}
$$

\subsubsection{Fuzzy comprehensive evaluation}

- The fuzzy comprehensive evaluation is gradually calculated from the low-level fuzzy evaluation formula to the top level to obtain the judgment result $B_{i}$; combined with the weight matrix $R_{i}$ obtained by the equation (8), the evaluation set $\mathrm{B}$ can be obtained.

$$
\begin{aligned}
& B_{i}=W_{i} R_{i} \\
& B=W R
\end{aligned}
$$

- Through the matrix operation, the final gray comprehensive evaluation value $\mathrm{Q}$ is obtained. For the calculated $\mathrm{Q}$ value and the actual situation of the project, considering the risk response measures and the cost and effect of the risk solution, the scientific selection solution.

$$
Q=B M
$$

\section{Case analysis}

For a power transmission and transformation project, a contractor with a design institute as the leader adopts the EPC mode to win the bid. According to the risk list and fuzzy evaluation model established in this paper, we will analyze the construction risk as follows:

- Judgment matrix $\mathrm{A}$ and $\mathrm{A}_{\mathrm{i}}$ of project-level risk and second-level risk weight can be obtained through the nine-degree comparison method, see Table 1-6.

Table 1. Risk indicator weight calculation.

\begin{tabular}{ccccccc}
\hline $\mathrm{A}$ & $U_{1}$ & $U_{2}$ & $U_{3}$ & $U_{4}$ & $U_{5}$ & $W$ \\
\hline$U_{1}$ & 1 & 2 & 3 & 4 & 2 & 0.371 \\
$U_{2}$ & $1 / 2$ & 1 & 2 & 3 & 2 & 0.239 \\
$U_{3}$ & $1 / 3$ & $1 / 2$ & 1 & 4 & 3 & 0.202 \\
$U_{4}$ & $1 / 4$ & $1 / 3$ & $1 / 4$ & 1 & 2 & 0.094 \\
$U_{5}$ & $1 / 2$ & $1 / 2$ & $1 / 3$ & $1 / 2$ & 1 & 0.094 \\
\hline
\end{tabular}

Table 2. Risk indicator weight calculation.

\begin{tabular}{ccccc}
\hline $\mathrm{A}_{1}$ & $U_{11}$ & $U_{12}$ & $U_{13}$ & $W$ \\
\hline$U_{11}$ & 1 & 2 & 4 & 0.571 \\
$U_{12}$ & $1 / 2$ & 1 & 2 & 0.286 \\
$U_{13}$ & $1 / 4$ & $1 / 2$ & 1 & 0.143 \\
\hline
\end{tabular}

Table 3. Risk indicator weight calculation.

\begin{tabular}{cccccc}
\hline $\mathrm{A}_{2}$ & $U_{21}$ & $U_{22}$ & $U_{23}$ & $U_{24}$ & $W$ \\
\hline$U_{21}$ & 1 & 3 & 2 & 3 & 0.444 \\
$U_{22}$ & $1 / 3$ & 1 & 2 & 3 & 0.255 \\
$U_{23}$ & $1 / 2$ & $1 / 2$ & 1 & 4 & 0.213 \\
$U_{24}$ & $1 / 3$ & $1 / 3$ & $1 / 4$ & 1 & 0.088 \\
\hline
\end{tabular}

Table 4. Risk indicator weight calculation.

\begin{tabular}{ccccc}
\hline $\mathrm{A}_{3}$ & $U_{31}$ & $U_{32}$ & $U_{33}$ & $W$ \\
\hline$U_{31}$ & 1 & $1 / 2$ & 4 & 0.359 \\
$U_{32}$ & 2 & 1 & 3 & 0.517 \\
$U_{33}$ & $1 / 4$ & $1 / 3$ & 1 & 0.124 \\
\hline
\end{tabular}

Table 5. Risk indicator weight calculation.

\begin{tabular}{ccccc}
\hline $\mathrm{A}_{4}$ & $U_{41}$ & $U_{42}$ & $U_{43}$ & $W$ \\
\hline$U_{41}$ & 1 & $1 / 3$ & 2 & 0.238 \\
$U_{42}$ & 3 & 1 & 4 & 0.625 \\
$U_{43}$ & $1 / 2$ & $1 / 4$ & 1 & 0.137 \\
\hline
\end{tabular}

Table 6. Risk indicator weight calculation.

\begin{tabular}{ccccc}
\hline $\mathrm{A}_{5}$ & $U_{51}$ & $U_{52}$ & $U_{53}$ & $W$ \\
\hline$U_{51}$ & 1 & $1 / 2$ & 3 & 0.348 \\
$U_{52}$ & 2 & 1 & 2 & 0.484 \\
$U_{53}$ & $1 / 3$ & $1 / 2$ & 1 & 0.168 \\
\hline
\end{tabular}

The results are brought into equation (5) for consistency test. The CR values are all less than the specified values, indicating that there is no problem with the consistency test.

- The scores of the second-level risk indicators by experts according to the risk level are shown in Table 7 below:

Table 7. Construction risk indicator score.

\begin{tabular}{ccccccccccc}
\hline aims & $U_{i}$ & $U_{i j}$ & expert 1 & expert 2 & expert 3 & expert 4 & expert 5 & expert 6 & expert 7 & expert 8 \\
\hline \multirow{2}{*}{$U_{1}$} & $U_{11}$ & 1 & 1 & 3 & 2 & 5 & 4 & 3 & 2 \\
& $U_{12}$ & 4 & 5 & 3 & 7 & 2 & 7 & 5 & 4 \\
& & $U_{13}$ & 1 & 1 & 6 & 3 & 5 & 5 & 6 & 1 \\
\cline { 2 - 10 } & $U_{21}$ & 3 & 4 & 1 & 5 & 5 & 4 & 0 & 4 \\
& $U_{2}$ & 2 & 1 & 4 & 3 & 5 & 2 & 5 & 3 \\
& $U_{22}$ & 1 & 1 & 1 & 3 & 5 & 2 & 0 & 4 \\
\hline
\end{tabular}




\begin{tabular}{ccccccccccc}
\hline & & $U_{24}$ & 0 & 0 & 0 & 2 & 1 & 1 & 1 & 0 \\
\cline { 2 - 10 } & $U_{3}$ & $U_{31}$ & 2 & 3 & 2 & 4 & 2 & 4 & 3 & 3 \\
& $U_{32}$ & 4 & 4 & 5 & 3 & 5 & 5 & 4 & 3 \\
& $U_{33}$ & 1 & 0 & 1 & 4 & 3 & 2 & 0 & 4 \\
\hline \multirow{2}{*}{$U_{4}$} & $U_{41}$ & 3 & 5 & 3 & 4 & 3 & 4 & 5 & 2 \\
& $U_{42}$ & 2 & 3 & 4 & 3 & 2 & 2 & 3 & 1 \\
& $U_{43}$ & 2 & 3 & 2 & 4 & 5 & 3 & 4 & 3 \\
\hline & $U_{51}$ & 4 & 1 & 4 & 3 & 4 & 2 & 4 & 4 \\
& $U_{5}$ & 1 & 4 & 3 & 3 & 5 & 4 & 3 & 4 \\
& $U_{52}$ & 3 & 3 & 5 & 3 & 5 & 5 & 4 & 3 \\
\hline
\end{tabular}

- Substituting the above parameters into the equation (7), the following results can be obtained by finishing.

$$
\begin{array}{ll}
r_{11}=(0.108,0.139,0.194,0.256,0.303) ; & r_{12}=(0.16,0.206,0.226,0.221,0.187) \\
r_{13}=(0.14,0.18,0.215,0.205,0.26) ; & r_{21}=(0.132,0.169,0.238,0.243,0.218) \\
r_{22}=(0.119,0.153,0.214,0.253,0.261) ; & r_{23}=(0.106,0.136,0.191,0.237,0.33) \\
r_{24}=(0.071,0.091,0.128,0.213,0.497) ; & r_{31}=(0.109,0.14,0.196,0.286,0.27) \\
r_{32}=(0.136,0.175,0.245,0.249,0.194) ; & r_{33}=(0.084,0.108,0.252,0.204,0.352) \\
r_{41}=(0.125,0.162,0.226,0.266,0.221) ; & r_{42}=(0.097,0.125,0.174,0.27,0.334) \\
r_{43}=(0.118,0.151,0.212,0.275,0.244) ; & r_{51}=(0.116,0.149,0.209,0.31,0.215) \\
r_{52}=(0.113,0.145,0.203,0.313,0.226) ; & r_{53}=(0.13,0.167,0.234,0.264,0.205)
\end{array}
$$

The first-level gray evaluation matrix of the firstlevel evaluation index $\left(U_{\mathrm{i}}\right)$ is calculated.

$$
\begin{gathered}
\left.R_{1}=\left(r_{11}, r_{12}, r_{13}\right)^{T} ; R_{2}=\underset{21}{ }, r_{22}, r_{31}, r_{41}\right)^{T} ; R_{3}=\left(r_{31}, r_{32}, r_{33}\right)^{T} ; R_{4}=\left(r_{41}, r_{42}, r_{43}\right)^{T} \\
R_{5}=\left(r_{51}, r_{52}, r_{53}\right)^{T}
\end{gathered}
$$

Through the processing of the above data, the fuzzy comprehensive evaluation calculation of the first-level evaluation index is carried out by using equation (9).

$$
\begin{aligned}
B_{1} & =W_{1} R_{1}=\left|\begin{array}{l}
0.571 \\
0.286 \\
0.143
\end{array}\right|^{T} \cdot\left|\begin{array}{ccccc}
0.108 & 0.139 & 0.194 & 0.256 & 0.303 \\
0.16 & 0.206 & 0.226 & 0.221 & 0.187 \\
0.14 & 0.18 & 0.215 & 0.205 & 0.26
\end{array}\right| \\
& =\left(\begin{array}{lllll}
0.1274 & 0.1640 & 0.2062 & 0.2387 & 0.2637
\end{array}\right)
\end{aligned}
$$

Similarly, it is available,

$$
\begin{aligned}
& B_{2}=W_{2} R_{2}=\left(\begin{array}{lllll}
0.1178 & 0.1510 & 0.2122 & 0.2416 & 0.2774
\end{array}\right) \\
& B_{3}=W_{3} R_{3}=\left(\begin{array}{lllll}
0.1199 & 0.1541 & 0.2283 & 0.2567 & 0.2409
\end{array}\right) \\
& B_{4}=W_{4} R_{4}=\left(\begin{array}{llllll}
0.1065 & 0.1374 & 0.1916 & 0.2697 & 0.2948
\end{array}\right) \\
& B_{5}=W_{5} R_{5}=\left(\begin{array}{lllll}
0.1169 & 0.1501 & 0.2103 & 0.3037 & 0.2186
\end{array}\right)
\end{aligned}
$$

From the above data, the comprehensive evaluation set can be finally obtained $\mathrm{B}$.

$$
\begin{aligned}
B=W R & =\left|\begin{array}{l}
0.371 \\
0.239 \\
0.202 \\
0.094 \\
0.094
\end{array}\right|^{T}\left|\begin{array}{llllll}
0.1274 & 0.1640 & 0.2062 & 0.2387 & 0.2637 \\
0.1178 & 0.1510 & 0.2122 & 0.2416 & 0.2774 \\
0.1199 & 0.1541 & 0.2283 & 0.2567 & 0.2409 \\
0.1065 & 0.1374 & 0.1916 & 0.2697 & 0.2948 \\
0.1169 & 0.1501 & 0.2103 & 0.3037 & 0.2186
\end{array}\right| \\
& =\left(\begin{array}{lllll}
0.1229 & 0.1582 & 0.2097 & 0.2514 & 0.2578
\end{array}\right)
\end{aligned}
$$


Finally, the fuzzy mathematics is used to perform the matrix operation, and the gray comprehensive evaluation

$$
\mathrm{Q}=\mathrm{BM}=\mid \begin{array}{lll}
0.1229 & 0.1582 & 0.2097
\end{array}
$$

Comparing this result with the above five evaluation factors, the overall risk of construction risk of this project is stable, between the "medium" and "lower" levels. There is a certain impact on the project, but it will not have a major impact. In this regard, the general contractor must take control and reduce the risk, transfer, and retention to prevent it from being converted to a higher level.

\section{Conclusion}

This paper analyzes the risk factors of the EPC power transmission and transformation project in the construction stage, and constructs a list of risk factors. The risk analysis is carried out by using the fuzzy comprehensive evaluation principle. I hope that the general contracting mode with design as the leader will play a certain enlightenment in the follow-up research in the field of power transmission and transformation. In addition, further analysis is needed for the analysis of the dynamic transformation of risks in the project.

\section{Acknowledgements}

The authors thank the State Grid Jibei Electric Power Company for supporting this work. This research was financially supported by Zhangjiakou Power Supply Company. We also thank the teachers of North China Electric Power University for their constructive comments during the review process.

\section{References}

1. Li, K. (2019) Research on project management of foreign power transmission and transform- ation projects. Engineering Construction and Design, 14:224-25.

2. Yan, H. (2017) Risk management of power engineering EPC general contracting project with design as the leader. Nanchang University, Nanchang.

3. Lin, Z. M., Zhang, W., Hou, X. L. (2015) Comprehensive evaluation of power transmission and transformation engineering management process system under EPC mode. Power System and Clean Energy, vol 31(10):89-93.

4. Zhang, L. M. (2015) Research on owner management interface of power transmission and transformation project based on EPC mode. North China Electric Power University, Beijing.

5. Zhang, C. J. (2015) Research on EPC General Contract Management of Power Transmission and Transformation Engineering in Electric Power Design Institute. North China Electric Power University, Beijing. value is determined by the equation (11).

$$
0.25140 .2578 \|\left. 97531\right|^{T}=4.274
$$

6. Zhong, Y. (2014) Research on EPC project management optimization of power transmission and transformation project in China. Enterprise Research, 18:40-46.

7. Lan, B., Yin, H. (2012) Research on project management of power transmission and transformation project under EPC mode. Project Management Technology, vol 10 (12): 25-29.

8. Xie, P. F. (2014) Research on the suitability of EPC mode for power transmission and transformation engineering of Group A. North China Electric Power University, Beijing. 